\title{
Saturable and Dispersive Parity-Time Symmetric Directional Coupler: a Transmission-Line Modelling Study
}

\author{
Sendy Phang ${ }^{1, *}$, Ana Vukovic ${ }^{1}$, Member, IEEE, Trevor M. Benson ${ }^{1}$, Senior Member, IEEE, Stephen \\ Creagh $^{2}$, Phillip Sewell ${ }^{1}$,Senior Member, IEEE, Gabriele Gradoni ${ }^{2}$ \\ ${ }^{1}$ George Green Institute for Electromagnetics Research, University of Nottingham, NG7 2RD, Nottingham, UK \\ ${ }^{2}$ School of Mathematical Sciences, University of Nottingham, NG7 2RD, Nottingham, UK \\ "e-mail: sendy.phang@nottingham.ac.uk
}

\begin{abstract}
In this paper, numerical modelling of Parity-Time (PT) coupled waveguides is reported. The PT coupled waveguide structure consists of two coupled slab waveguides based on GaAs material with gain/loss material parameter models, including both dispersion and saturation. The numerical model used analyses the impact of dispersion and saturation on the eigenmode extracted by a curve fitting approach. The results show that the presence of saturation may prohibit the appearance of the threshold point above which the PT system becomes unstable.
\end{abstract}

Keywords: parity-time waveguides, saturation model, TLM

\section{INTRODUCTION}

Optical waveguides with balanced gain and loss mimicking the parity-time (PT) symmetric systems have been the subject of intense investigation in the last few years [1]. Several different PT structures have been investigated, such as PT Bragg gratings [2-4], PT couplers [5-9], PT whispering gallery resonators [10] and PT lattices [11-14]. All PT structures are characterised by a non-symmetric response [2,3,15-17], i.e. they depend on the port of the input incidence. Although these investigations have been mainly theoretical studies using a coupled mode method [3,18,19] and ideal gain/loss model, i.e. no saturation and dispersion, several notable experimental observations have supported their theoretical predictions [10,12,14,15,20]. Another notable property of PT devices is the existence of a threshold point separating complex modes from purely real ones $[5,8,19]$.

In this paper, we explore the impact of saturation and dispersion on the behaviour of coupled PT waveguides using a time-domain numerical method. Recently, the impact of dispersion has been reported to be capable of reducing the bandwidth of unidirectional invisibility of PT Bragg gratings [4]. The impact of saturation on nonlinear PT Bragg gratings has been analysed and shown to be able to significantly undermine the interplay between the PT and nonlinear behaviours. This paper extends this analysis to PT coupled waveguides and, in particular, explores how saturation impacts eigenmode behaviour and the threshold gain/loss point. A curve fitting approach is adopted in order to extract the complex eigenmodes from a spatial Fourier transform and thus alleviate the resolution problem that is associated with the finite propagation length.

Throughout this paper, time-domain modelling is done using Transmission-Line Modelling (TLM) method. The TLM is a flexible time-stepping numerical technique that has been extensively characterised and used over many years $[21,22]$. However any time-domain method, including the Finite-Difference Time-Domain (FDTD), could be employed for this purpose and we refer the reader to the extensive literature for further details of both of these algorithms [23,24].

\section{PARITY-TIME (PT) COUPLED WAVEGUIDE AND MATERIAL MODEL}

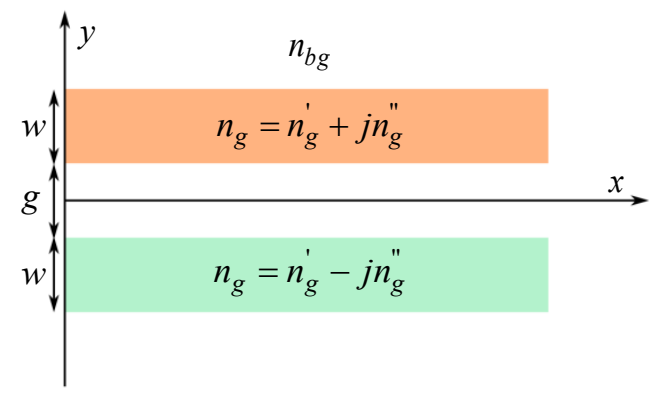

Fig. 1 Schematic illustration of PT directional coupled waveguide 
In this section, the PT coupled waveguide is described together with the model for gain/loss dispersion and saturation. The approach of extracting PT eigenmodes from time domain simulations is also described. A PT directional coupler is illustrated in Fig. 1 and comprises of two slab waveguides of width $w$, separated by a gap $g$ and embedded in a background medium $n_{b g}$. The refractive index of the waveguide core is denoted by $n_{g}^{\prime} \pm j n_{g}^{\prime}$ where "+" denotes a gain and "-"” a lossy medium.

A dispersive and saturable gain/loss model exhibiting a homogenous broadening with a Lorentzian profile [25] is implemented in the TLM method as,

$$
|\alpha|(\omega, I)=\Omega(I)\left(\frac{\alpha_{0} / 2}{1+j\left(\omega-\omega_{0 \sigma}\right) \tau}+\frac{\alpha_{0} / 2}{1+j\left(\omega+\omega_{0 \sigma}\right) \tau}\right),
$$

where gain/loss per unit length is related to the imaginary part of the refractive index by $\alpha_{0}=(\omega / c) n_{g}^{\prime \prime}, \omega_{0 \sigma}$ denotes the atomic transitional angular frequency and $\tau$ is the dipole relaxation time parameter. The intensity independent function $\Omega$ is the gain/loss saturation factor defined by

$$
\Omega(I)=\frac{1}{1+I / I_{s}}
$$

where $I$ and $I_{s}$ denote the input signal and saturation intensity respectively. The saturation factor $\Omega$ describes the gain/loss saturation level, and varies between $0<\Omega<1$, with $\Omega \rightarrow 0$ indicating high and $\Omega \rightarrow 1$ indicating low saturation level, respectively.

\subsection{Effective Refractive Index Extraction by curve fitting}

As a time-domain technique, the TLM method simulates the field propagation in both the space and time domains. In order to extract accurately the real part of effective refractive indices, a good resolution is needed in spatial $k$-space, which in turn requires that the simulation is performed along a very long waveguide. Although it is possible to extract the real part of the effective refractive index, obtaining the imaginary part of the effective index is not as direct. In order to deal with a finite propagation length, a curve fitting technique is used to solve for unknown complex eigenmodes. A similar approach in the time domain has been used in [26] to extract complex eigenfrequencies of a whispering gallery resonator and is here adapted for spatial eigenmode extraction.

The field propagating along the $x$ direction, with a spatial mode distribution $\varphi$ in the $y$ direction, can be expressed as,

$$
\psi(x, y)=\sum_{m} \varphi_{m}(y) e^{j \beta_{x, m} x}
$$

Where the propagation constant $\beta_{x, m}=k_{0}\left(n_{m}^{\prime} \pm j n_{m}^{\prime \prime}\right)$ with $n_{m}^{\prime}$ and $n_{m}^{\prime \prime}$ are the real and imaginary parts of the effective index of eigenmodes $m$ and $k_{0}=\frac{2 \pi}{c} f$ with $f$ the operating frequency. The theoretical spatial Fourier transform along the propagating direction of the field in equation (3) is,

$$
\Psi\left(\beta_{x}, y\right)=\sum_{m} \varphi_{m}(y) \int_{-\infty}^{\infty} e^{j k_{0}\left(n_{m}^{\prime}-j n_{m}^{\prime \prime}\right) x} e^{-j k_{0} n x} d x
$$

The analytical Fourier transform in $k$-space along a finite length $x=L$ is,

$$
\Psi\left(\beta_{x}, y\right) \approx \sum_{m} \varphi_{m}(y) \int_{0}^{L} e^{j k_{0}\left(n_{m}^{\prime}-j n_{m}^{\prime \prime}\right) x} e^{-j k_{0} n x} d x=\sum_{m} \varphi_{m}(y)\left(\frac{1-e^{-j k_{0} L\left(n-n_{m}^{\prime}-j n_{m}^{\prime \prime}\right)}}{k_{0}\left(n_{m}^{\prime \prime}-j n_{m}^{\prime}\right)}\right)
$$

In order to extract unknown values $n_{m}^{\prime}$ and $\pm n_{m}^{\prime \prime}$ a curve in the form of absolute value of equation (5) is fitted to the discrete data obtained from the numerical TLM simulation. 


\section{RESULTS AND DISCUSSIONS}

In this section the PT directional coupler is modelled using the TLM method. The accuracy of the curve fitting for solving waveguide eigenmodes is first tested on the case of a PT waveguide with non-saturable gain/loss i.e. $\Omega=1$ and then applied to the case of saturable gain/loss.

The PT directional coupler in this paper has the following parameters: the core refractive index is $n_{g}^{\prime}=3.5$, background refractive index is $n_{b g}=1.3$, waveguide width is $w=0.15 \mu \mathrm{m}$ and waveguide separation $g=0.15$ $\mu \mathrm{m}$. The length of the PT waveguide is $40 \mu \mathrm{m}$. The gain/loss parameters are $\omega_{0 \sigma}=2116.5 \mathrm{rad} / \mathrm{ps}$ and $\tau=0.1$ ps. The TLM simulation has the following parameters: the spatially mesh has $\Delta x=\Delta y=7.5 \times 10^{-3} \mu \mathrm{m}$ and was run for 260000 time steps, which ensured that the optical signal reached steady state. The loss waveguide is initially excited with the analytical mode profile. In this paper the TM polarisation is considered.

\subsection{Unsaturated gain/loss system $(\Omega=1)$}

Fig. 2(a) shows the magnetic field $H_{z}$ of the propagating TM mode in space as it propagates along the $x$ direction. Fig. 2(a) shows that the wave is coupled from the lossy waveguide to the gain waveguide. It is noted that when a total coupling occurs, a maximum field is observed in one channel followed by a minimum in the other, thus displaying the typical sinusoidal profile of power coupling between two waveguides. Fig. 2(b) shows the $k$-space plot of the magnetic field $H_{z}$, obtained by spatial Fourier transformations in both the $x$ and $y$ directions of Fig. 2(a). Fig. 2(b) shows the presence of two guided modes propagating in the $x$ direction, which make up a supermode in Fig. 2(a). Ideally two distinct discrete lines would be shown if the Fourier transformations were performed in an infinite domain (equation (4)). However, since the Fourier transformations are performed in a finite domain these two mode lines spread and are accompanied by a continuous trail of side lobes as shown in Fig.2(c). Fig.2(c) is obtained by projecting, i.e. summing all modes in Fig.2(b), to the horizontal axis. Fig. 2(c) depicts two distinct peaks, which correspond to the two guiding TM modes, the discrete points denoting the direct projections of Fig. 2(b) while the solid line is obtained by curve fitting the discrete point by equation (5). The MATLAB curve fitting toolbox is used for curve fitting purposes.
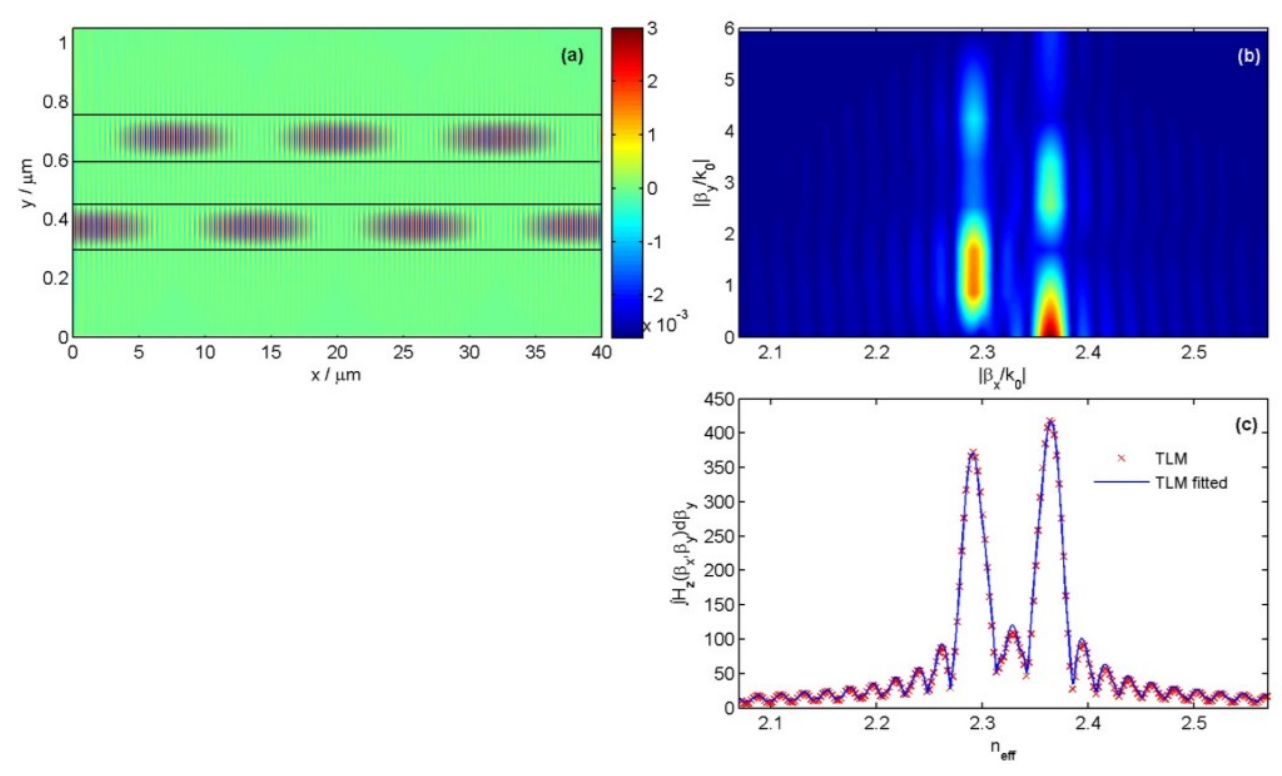

Fig. 2(a) $H_{z}$ field profile in $x$-y space domain, (b) $k$-space plot obtained by spatial Fourier transformation of (a) in both directions and (c) the projected $k$-space plot on the horizontal axis.

To investigate the impact of gain/loss on the effective refractive index, the (a) real and (b) imaginary part of the effective indices are plotted in Fig. 3(a,b) as a function of the gain/loss parameter $\alpha_{0}$. As the saturation factor $\Omega=1$ this is the case of non-saturable gain and loss for which an analytical solution exists and is also plotted in Fig. 3. Analytical solutions are for the threshold point $\alpha_{t r}=2400 \mathrm{~cm}^{-1}$, below which the eigenmodes are purely real and above which the eigenmodes are complex conjugate. The discrete points in Fig. 3 show the effective refractive indices obtained using the TLM and the curve fitting technique. The real part of the refractive 
index (Fig. 3(a)) displays a similar trend as the one calculated by the analytical method but is slightly higher in value. This discrepancy can be explained by the presence of numerical dispersion. Fig. 3(b) shows a good agreement between the imaginary parts of the effective refractive index calculated by the TLM method and by the analytical method, with small a discrepancy occurring around the threshold point where the two guided modes are about to coalesce. It is noted that during the fitting process, the minimum goodness (R-parameter) of the fitted curve occurs for gain/loss parameters close to the threshold point and is around $98 \%$.
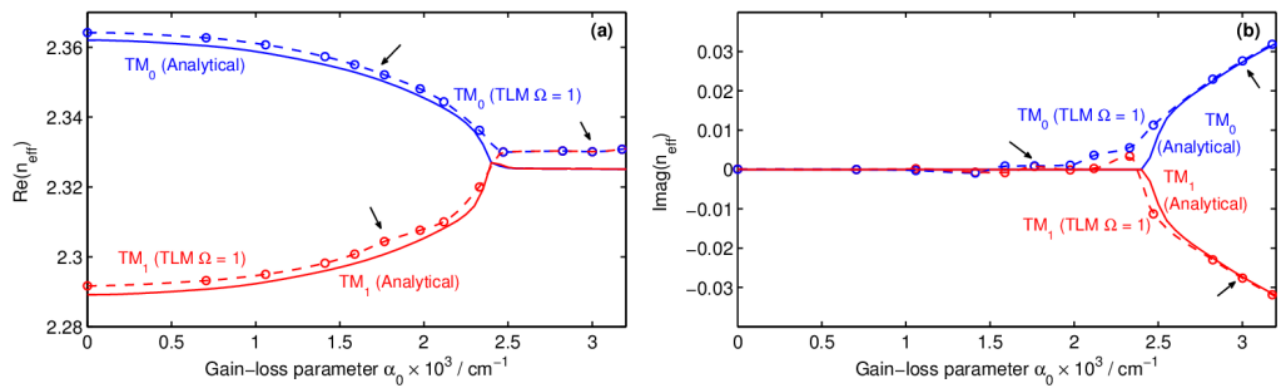

Fig. 3 Comparison of (a) the real part and (b) the imaginary part of the effective refractive index of the PT coupled waveguide obtained by the TLM and curve fitting method (dashed lines) and the analytical method.

Fig. 4(a,b) shows the magnetic field $H_{z}$ distribution in $x-y$ space domain for gain/loss the parameter of (a) $\alpha_{0}=1765 \mathrm{~cm}^{-1}$, below the threshold point and (b) above the threshold point for $\alpha_{0}=3000 \mathrm{~cm}^{-1}$, as marked with arrows in Fig. 3. When operated below the threshold point, Fig. 4(a) shows that the coupling length is longer than that compared to the case of no gain/loss in Fig. 2(a). Fig. 2(a) shows 3 complete coupling cycles compared to only 2 cycles in Fig. 4(a) for the same propagation length of $40 \mu \mathrm{m}$. It is also noted that the field distribution profile is asymmetric due to the presence of gain and loss. The $H_{z}$ profile for operation above the threshold point is displayed in Fig. 4(b), where the field in both channels is exponentially growing and where the growing mode dominates. Fig. 4(c,d) shows the projected $k$-space plot of the magnetic field shown in Fig. 4(a,b), for the operation of (c) below and (d) above the threshold point. Fig. 4(c) shows two distinct guiding modes that correspond to the case of Fig. 4(a), whilst Fig. 4(d) has a single peak corresponding to a single mode existing above the threshold point as predicted by Fig. 3(a,b).
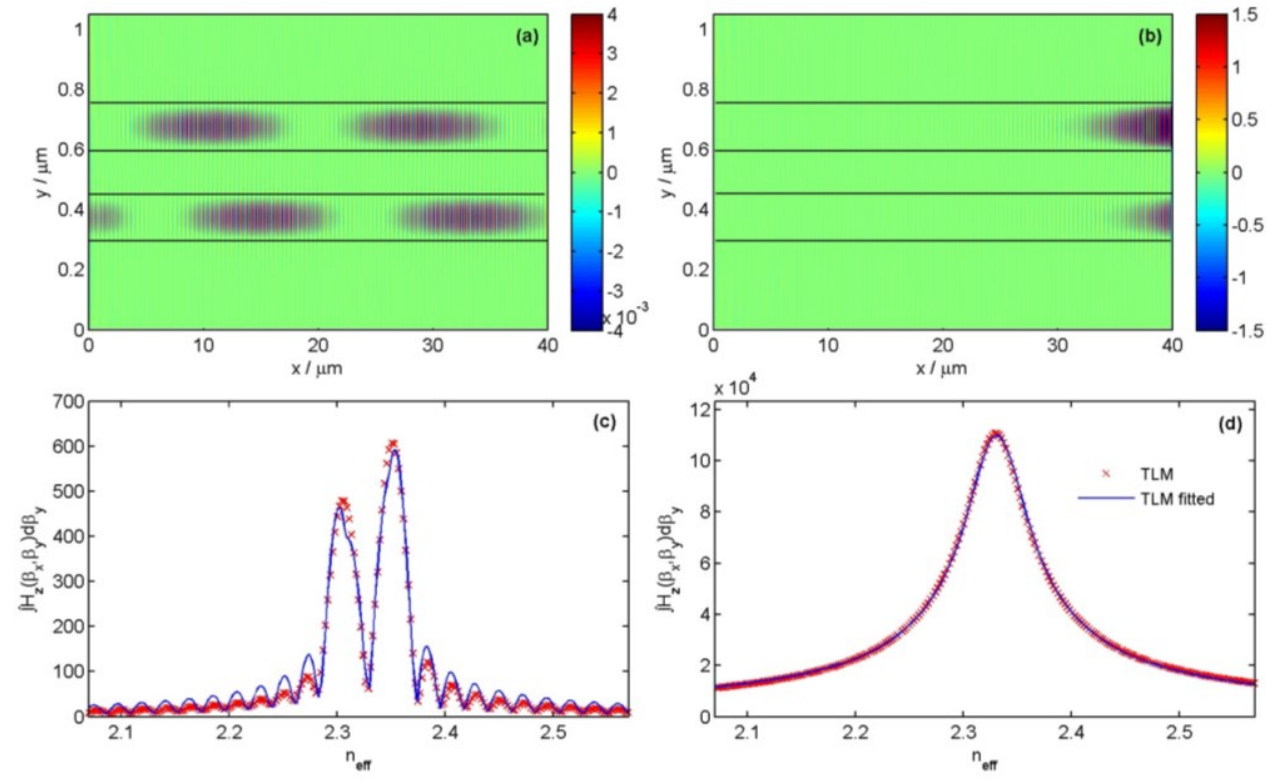

Fig. $4(a, b) H_{z}$ field profile in $x$-y space for operation (a) below threshold point $\alpha_{0}=1765 \mathrm{~cm}^{-1}$ and (b) above threshold point $\alpha_{0}=3000 \mathrm{~cm}^{-1}$. The projected $k$-space plots for operation (c) below the threshold point $\alpha=1765 \mathrm{~cm}^{-1}$ and (d) above threshold point $\alpha=3000 \mathrm{~cm}^{-1}$. Gain/loss is considered linear $\Omega=1$. 


\subsection{Saturable gain/loss system}

In this section the impact of gain/loss saturation on the eigenmode solutions of the PT directional coupler is analysed. For this purpose an incident signal with an intensity of $I=3.64 \times 10^{7} \mathrm{Wm}^{-2}$ is launched in the lossy waveguide. The gain/loss has a saturation intensity $I_{s}=65.2 \times 10^{7} \mathrm{Wm}^{-2}$. The TLM parameters are as given in section 3.1 .
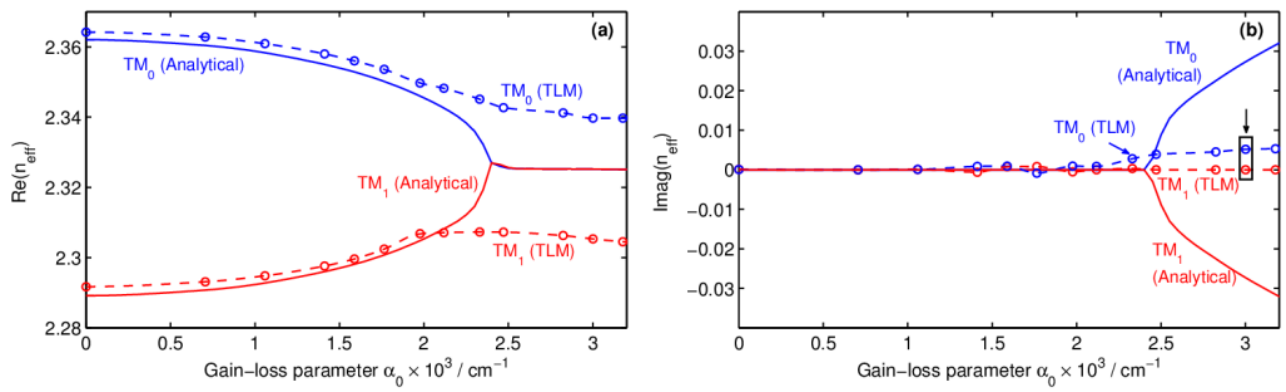

Fig. 5 Comparison of (a) real and (b) imaginary part of the effective index as a function of gain/loss parameter obtained using the TLM method and curve fitting for a case of a saturable gain/loss and analytical solution with no gain/loss saturation.

Fig. 5(a,b) shows the real and imaginary part of the effective refractive index as a function of the gain/loss parameter $\alpha_{0}$, obtained by the TLM and curve fitting together with the analytical solution of a linear system $(\Omega=1)$. Compared with the ideal case, Fig. 5 shows that in the case of a saturable gain/loss PT waveguide the two eigenmodes never coalesce and hence there is no threshold point. It can be explained that as the optical intensity in the waveguide increases, the effective gain/loss decreases due to saturation. Consequently, as shown in Fig. 5(b), the guided modes have small imaginary effective index (gain/loss) with a more strongly growing mode.
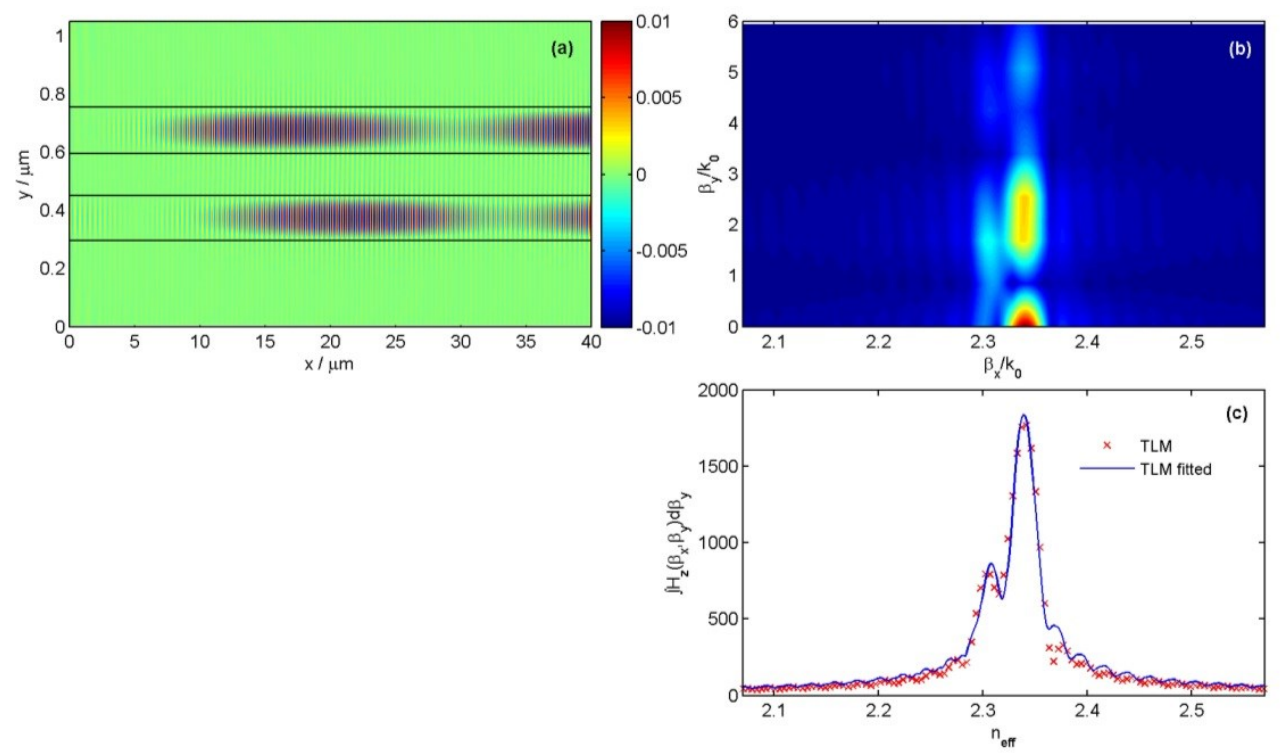

Fig. 6(a) Magnetic field $H_{z}$ profile distribution in space domain, (b) $k$-space plot obtained by spatial Fourier transformation in both directions of (a), (c) the projected $k$-space plot shown in (b) into the horizontal axis. Operated with gain/loss parameter of $\alpha=3000 \mathrm{~cm}^{-1}$ and saturation intensity of $I_{s}=65.2 \times 10^{7} \mathrm{Wm}^{-2}$.

Fig. 6(a) shows the- $H_{z}$ distribution in the $x-y$ domain for $\alpha_{0}=3000 \mathrm{~cm}^{-1}$ as marked in Fig. 5. Fig. 6(a) shows coupling from both channels. This is in contrast to the same operational point for linear gain/loss profile (Fig. 4(b)) where the field is exponentially growing. Fig. 6(b) shows the $k$-space plot of the field profile distribution in 
Fig. 6(a). Fig. 6(b) shows two guided modes with a distinct line at around $n_{\text {eff }}=2.34$ and a degenerating mode at around $n_{\text {eff }}=2.30$. Fig. 6(c) shows the projection of the $k$-space plot shown in Fig. 6(b) calculated by the TLM method (discrete points) and the fitted curve by equation (5). Fig. 6(c) shows the presence of two guided modes which are very close compared to the case of no gain/loss (Fig. 2(c)) and also that the growing mode dominates the field profile.

\section{CONCLUSIONS}

This paper analysed the impact of the saturable and dispersive gain/loss material model on the eigenmode solutions of a PT directional coupler using the TLM method and curve fitting technique to extract the complex eigenmode solutions. Good agreement is obtained when the results obtained using the TLM and the curve fitting technique are compared against the analytical solutions for the case of no gain/loss saturation. The results show that the presence of saturation may prohibit the appearance of the threshold point, resulting in a stable system.

\section{REFERENCES}

1. C. M. Bender, S. Boettcher, and P. N. Meisinger, "PT-symmetric quantum mechanics," Journal of Mathematical Physics 40, 2201 (1999).

2. Z. Lin, H. Ramezani, T. Eichelkraut, T. Kottos, H. Cao, and D. N. Christodoulides, "Unidirectional Invisibility Induced by PT-Symmetric Periodic Structures," Physical Review Letters 106, 213901 (2011).

3. M. Kulishov, J. M. Laniel, N. Bélanger, J. Azaña, and D. V Plant, "Nonreciprocal waveguide Bragg gratings," Optics Express 13, 3068-78 (2005).

4. S. Phang, A. Vukovic, H. Susanto, T. M. Benson, and P. Sewell, "Impact of dispersive and saturable gain/loss on bistability of nonlinear parity-time Bragg gratings.," Optics letters 39, 2603-6 (2014).

5. L. Chen, R. Li, N. Yang, D. Chen, and L. Li, "Optical Modes in PT-Symmetric Double-Channel Waveguides," arXiv preprint arXiv: 1202.2956 (2012).

6. H. Nolting, G. Sztefka, and J. Čtyroký, "Wave Propagation in a Waveguide with a Balance of Gain and Loss," in Integrated Photonics Research (OSA, 1996), pp. 76-80.

7. M. Greenberg and M. Orenstein, "Unidirectional complex grating assisted couplers.," Optics Express 12, 4013-8 (2004).

8. J. Čtyroký, "Dispersion properties of coupled waveguides with loss and gain: a full-vectorial analysis," Optical and Quantum Electronics (2014).

9. J. Čtyroký, V. Kuzmiak, and S. Eyderman, "Waveguide structures with antisymmetric gain/loss profile," Optics Express 18, 21585-21593 (2010).

10. B. Peng, Ş. K. Özdemir, F. Lei, F. Monifi, M. Gianfreda, G. L. Long, S. Fan, F. Nori, C. M. Bender, and L. Yang, "Parity-time-symmetric whispering-gallery microcavities," Nature Physics 10, 1-33 (2014).

11. S. Nixon, L. Ge, and J. Yang, "Stability analysis for solitons in PT-symmetric optical lattices," Physical Review A 85, 023822 (2012).

12. A. Regensburger, C. Bersch, M.-A. Miri, G. Onishchukov, D. N. Christodoulides, and U. Peschel, "Paritytime synthetic photonic lattices.," Nature 488, 167-71 (2012).

13. K. G. Makris, R. El-Ganainy, and D. N. Christodoulides, "Beam Dynamics in PT Symmetric Optical Lattices," Physical Review Letters 100, 103904 (2008).

14. A. Regensburger, M. Miri, and C. Bersch, "Observation of Defect States in PT-Symmetric Optical Lattices," Physical Review Letters (2013).

15. C. E. Rüter, K. G. Makris, R. El-Ganainy, D. N. Christodoulides, M. Segev, and D. Kip, "Observation of parity-time symmetry in optics," Nature Physics 6, 192-195 (2010).

16. T. Kottos, "Optical physics: Broken symmetry makes light work," Nature Physics 6, 166-167 (2010).

17. S. Phang, A. Vukovic, H. Susanto, T. M. Benson, and P. Sewell, "Ultrafast optical switching using paritytime symmetric Bragg gratings," Journal of the Optical Society of America B 30, 2984 (2013).

18. R. El-Ganainy, K. G. Makris, D. N. Christodoulides, and Z. H. Musslimani, "Theory of coupled optical PTsymmetric structures," Optics Letters 32, 2632 (2007).

19. H. Benisty, A. Degiron, A. Lupu, A. De Lustrac, S. Forget, M. Besbes, G. Barbillon, A. Bruyant, S. Blaize, and G. Lérondel, "Implementation of PT symmetric devices using plasmonics : principle and applications," Optics Express 19, 3567-3578 (2011).

20. L. Feng, Y.-L. Xu, W. S. Fegadolli, M.-H. Lu, J. E. B. Oliveira, V. R. Almeida, Y.-F. Chen, and A. Scherer, "Experimental demonstration of a unidirectional reflectionless parity-time metamaterial at optical frequencies.," Nature Materials 12, 108-13 (2013).

21. W. J. R. Hoefer, "The Transmission-Line Matrix Method--Theory and Applications," IEEE Transactions on Microwave Theory and Techniques 33, 882-893 (1985).

22. C. Christopoulos, The Transmission-Line Modeling Method TLM (IEEE Press, 1995). 
23. M. Krumpholz, C. Huber, and P. Russer, "A field theoretical comparison of FDTD and TLM," IEEE Transactions on Microwave Theory and Techniques 43, 1935-1950 (1995).

24. M. N. O. Sadiku and C. N. Obiozor, "A comparison of finite difference time-domain (FDTD) and transmission-line modeling (TLM) methods," in Proceedings of the IEEE SoutheastCon 2000. "Preparing for The New Millennium" (Cat. No.00CH37105) (IEEE, n.d.), pp. 19-22.

25. S. C. Hagness, R. M. Joseph, and A. Taflove, "Subpicosecond electrodynamics of distributed Bragg reflector microlasers: Results from finite difference time domain simulations," Radio Science 31, 931-941 (1996).

26. A. Vukovic, P. Sewell, T. M. Benson, and H. G. Dantanarayana, "Resonant frequency and Q factor extraction from temporal responses of ultra-high Q optical resonators," IET Science, Measurement \& Technology 8, (2014). 